\title{
A formalization of one of the main claims of "OpenBiodiv: A knowledge graph for literature-extracted linked open data in biodiversity science" by Penev et al. $2019^{1}$
}

\author{
Mariya Dimitrova \\ Bulgarian Academy of Sciences, Bulgaria and Pensoft Publishers, Bulgaria \\ E-mail: m.dimitrova@pensoft.net; ORCID: https://orcid.org/0000-0002-8083-6048 \\ Editor: Cristina-Iulia Bucur (https://orcid.org/0000-0002-7114-6459) \\ Review comments from: Tobias Kuhn (https://orcid.org/0000-0002-1267-0234); Ricardo Usbeck \\ (https://orcid.org/0000-0002-0191-7211); Cristina-Iulia Bucur (https://orcid.org/0000-0002-7114-6459)
}

Received 7 July 2021

Accepted 3 December 2021

\begin{abstract}
Penev et al. claimed in previous work that triples in the OpenBiodiv knowledge graph releases are generally semantic triples extracted from biodiversity literature. We present here a formalization of that claim, stating that all things of class "triple in OpenBiodiv knowledge graph" that are in the context of a thing of class "release of OpenBiodiv knowledge graph" generally have a relation of type "is same as" to a thing of class "semantic triple extracted from biodiversity literature" in the same context.
\end{abstract}

Keywords: release of OpenBiodiv knowledge graph, triple in OpenBiodiv knowledge graph, semantic triple extracted from biodiversity literature

\section{Introduction}

Penev et al. [2] state that "OpenBiodiv encompasses data extracted from more than 5000 scholarly articles published by Pensoft and many more taxonomic treatments extracted by Plazi from journals of other publishers. The data from both sources are converted to Resource Description Framework (RDF) and integrated in a graph database using the OpenBiodiv-O ontology and an RDF version of the Global Biodiversity Information Facility (GBIF) taxonomic backbone.”. We present here a formalization of the main scientific claim from this quote by using a semantic template called the super-pattern [1].

\footnotetext{
${ }^{1}$ As RDF/nanopublication: http://purl.org/np/RAMgThXW6xx8QiPmW9VhVuxWCN2ZWe-pmxDcFfdx_A7z0 


\section{Formalization}

Our formalization looks as follows:

\author{
CONTEXT-CLASS ("in the context of all..."): \\ SUBJECT-CLASS ("things of type..."): \\ QUALIFIER: \\ RELATION-TYPE ("have a relation of type..."): \\ OBJECT-CLASS ("to things of type..."):
}

\author{
release of OpenBiodiv knowledge graph \\ triple in OpenBiodiv knowledge graph \\ generally \\ is same as \\ semantic triple extracted from biodiversity literature
}

In the context class we use a new minted class "release of OpenBiodiv knowledge graph" that is a subclass of "software release" (Q20631656) from Wikidata and has a related class a new minted class "OpenBiodiv knowledge graph" that is a subclass of the "knowledge graph" (Q33002955) class from Wikidata. In the subject class, we use a new minted class "triple in OpenBiodiv knowledge graph" that is related to a new minted class "OpenBiodiv knowledge graph" that is a subclass of the "knowledge graph" (Q33002955) class from Wikidata. In the object class we minted a new class "semantic triple extracted from biodiversity literature" that is related to a new minted class "OpenBiodiv knowledge graph" that is a subclass of the "knowledge graph" (Q33002955) class from Wikidata.

\section{RDF code}

This is our formalization as a nanopublication in TriG format:

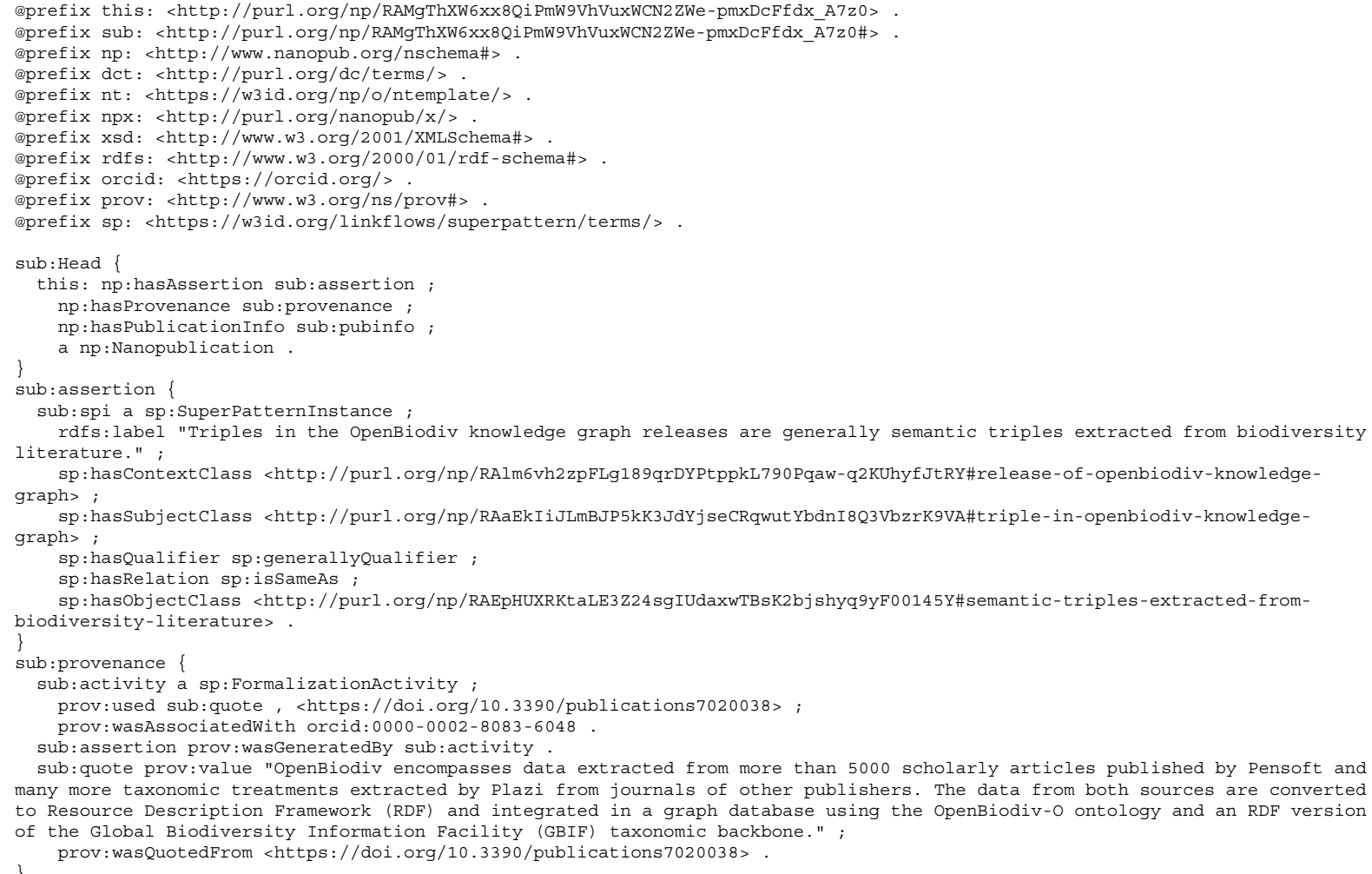




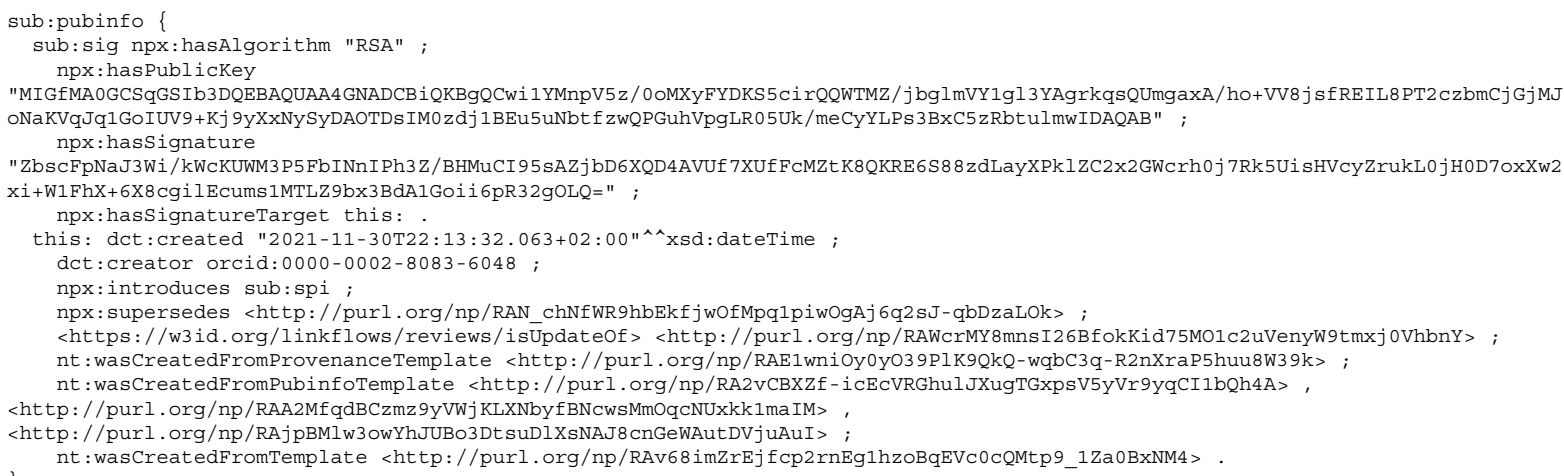

The following nanopublications introduce the newly minted classes in TriG format. This is the class definition of "release of OpenBiodiv knowledge graph":

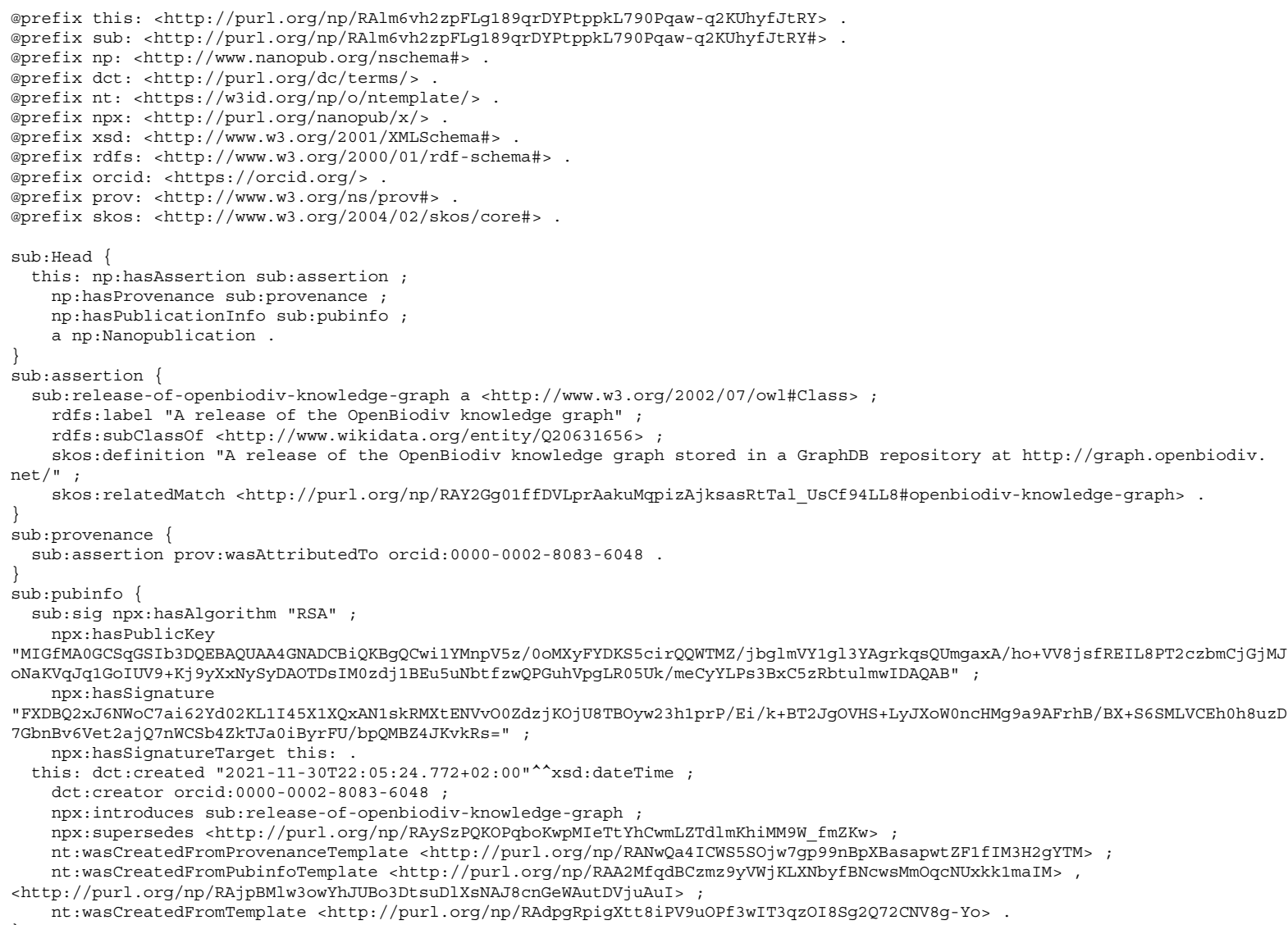

\section{This is the class definition of "triple in OpenBiodiv knowledge graph":}

@prefix this: <http://purl.org/np/RAaEkIiJLmBJP5kK3JdYjseCRqwutYbdnI803VbzrK9VA> @prefix sub: <http://purl org/np/RAaEkIiJLmBJP5kK3JdYj seCRgwutYbdnI8Q3VbzrK9VA\#> @prefix np: <http://www. nanopub.org/nschema\#>

@prefix dct: <http://purl.org/dc/terms/>

@prefix nt: <https://w3id.org/np/o/ntemplate/> . 


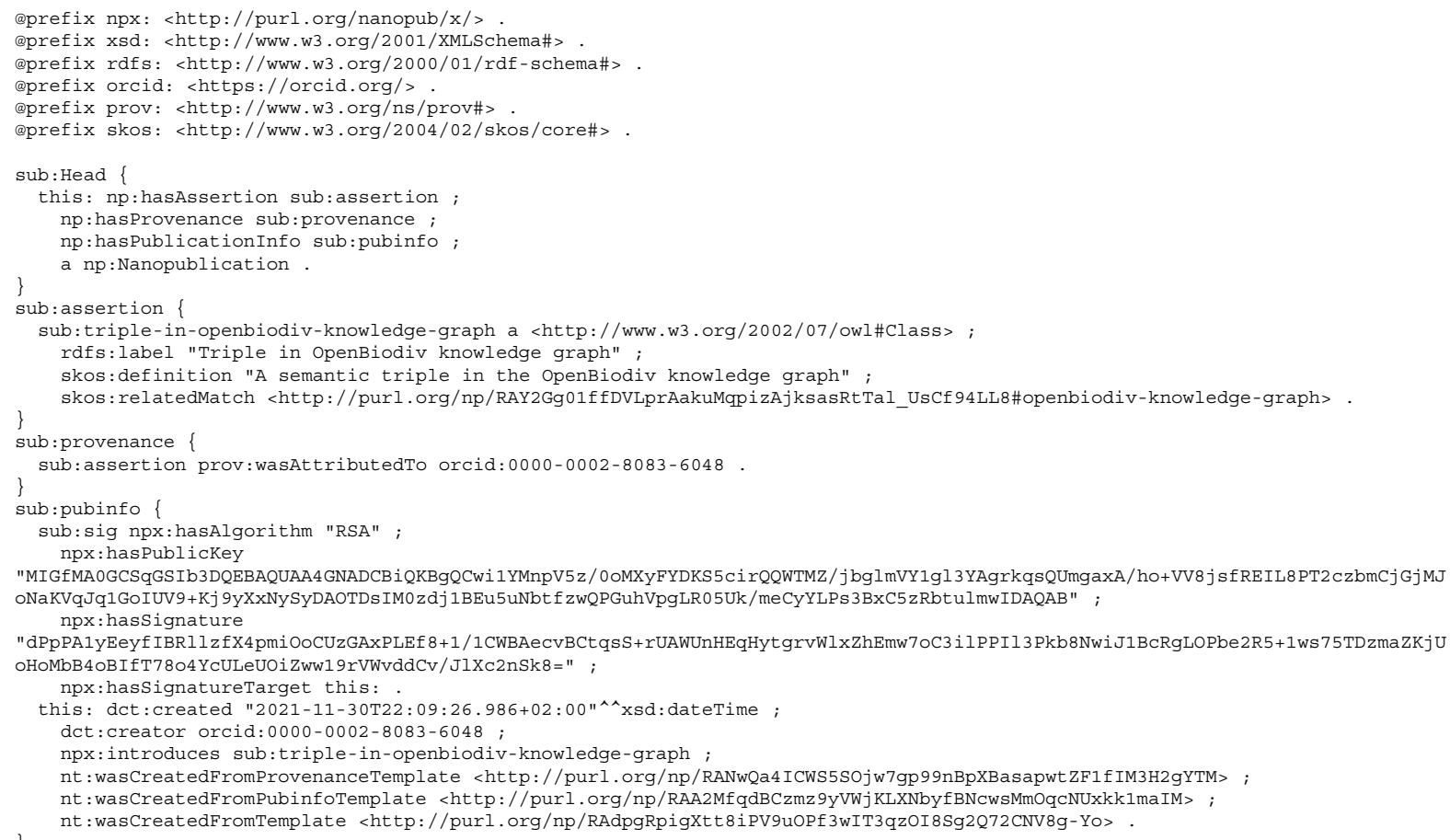

\section{This is the class definition of "semantic triple extracted from biodiversity literature":}

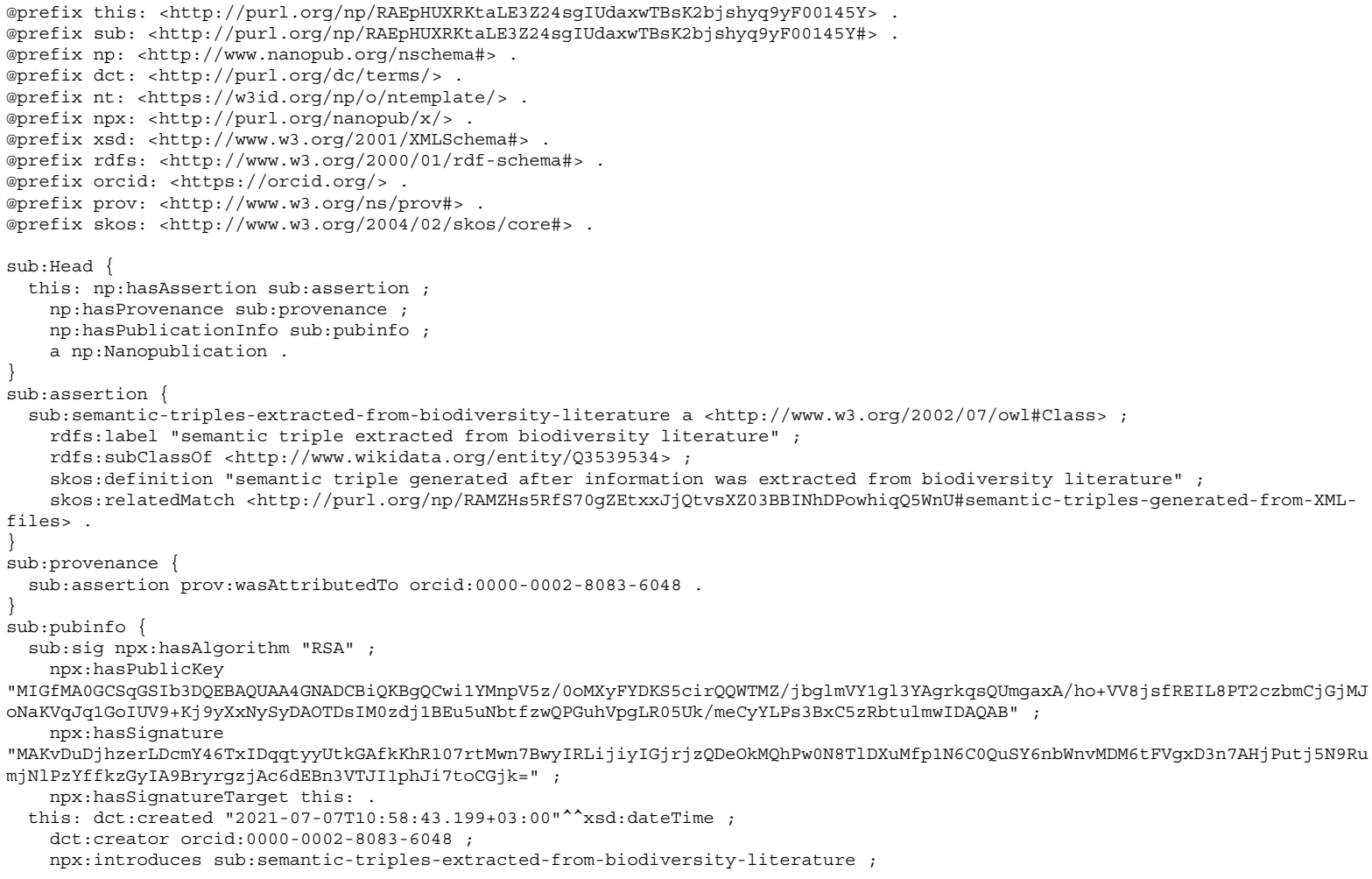


nt:wasCreatedFromProvenanceTemplate <http://purl.org/np/RANwQa4ICWS5SOjw7gp99nBpXBasapwtZF1f IM3H2gYTM> nt: wasCreatedFromPubinfoTemplate <http://purl.org/np/RAA2MfgdBCzmz9yVWj KLXNbyfBNCwSMmOgcNUxkk1maIM> \} nt: wasCreatedFromTemplate <http://purl.org/np/RAdpgRpigXtt8iPV9uOPf3wIT3qzOI8Sg2Q72CNV8g-Yo>

This is the class definition of "OpenBiodiv knowledge graph":

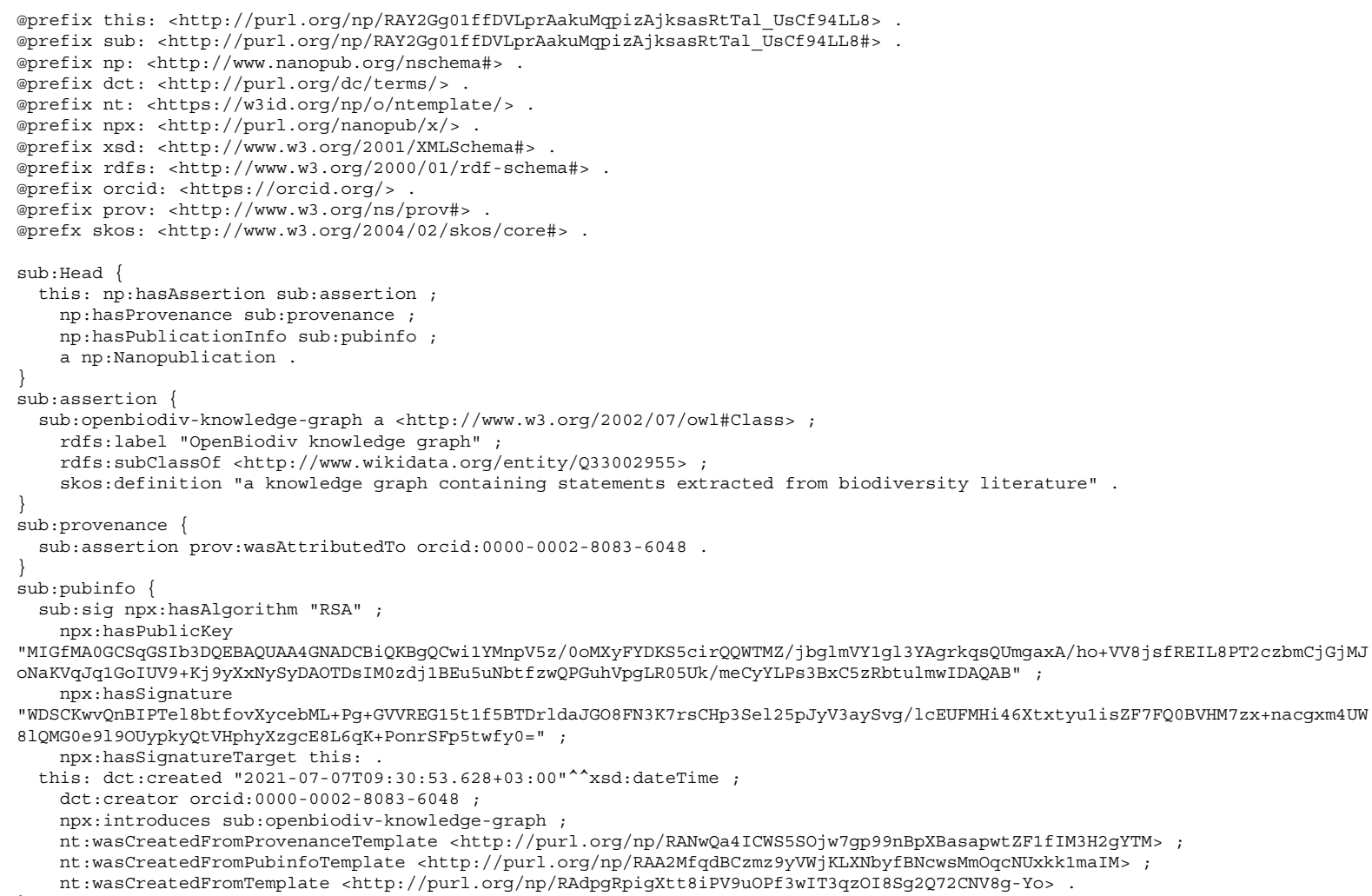

\section{Funding}

This research received funding from the European Union's Horizon 2020 research and innovation programme under the Marie Sklodowska-Curie grant agreement IGNITE (No. 764840).

\section{References}

[1] C.I. Bucur, T. Kuhn, D. Ceolin and J. van Ossenbruggen, Expressing high-level scientific claims with formal semantics, Proceedings of the 11th Knowledge Capture Conference 2021. doi:10.1145/3460210.3493561.

[2] L. Penev, M. Dimitrova, V. Senderov, G. Zhelezov, T. Georgiev, P. Stoev, Simov and K. OpenBiodiv, A Knowledge Graph for Literature-Extracted Linked Open Data in Biodiversity Science, Vol. 7, Publications, 2019, p. 38. doi:10.3390/ publications7020038. 